\title{
In vitro Evaluation of Some Fungicides against Indian Isolates of Sclerotium rolfsii Sacc. [Teleomorph: Athelia rolfsii (Curzi) Tu \& Kimbrough]
}

\author{
R.K. Sharma ${ }^{1^{*}}$ and I.U. Dhruj ${ }^{2}$ \\ ${ }^{1}$ Department of Plant Pathology, Agricultural Research Station, S. D. Agricultural University, \\ Ladol, Gujarat, India \\ ${ }^{2}$ Office of the Directorate of Research, Junagadh Agricultural University, Junagadh, \\ Gujarat, India
}

*Corresponding author

\section{Keywords}

Sclerotium rolfsii, Isolates, Fungicides, in vitro evaluation

Article Info

Accepted:

12 October 2018

Available Online:

10 November 2018

\section{A B S T R A C T}

In vitro evaluation of some fungicides (systemic and non-systemic) against twenty different Indian isolates of Sclerotium rolfsii were tested on potato dextrose agar medium by using poisoned-food technique and per cent growth inhibition was recorded at ranging from 125, 250 and 500 ppm for systemic; and 500, 1000 and 2000 ppm for non-systemic, respectively. For fungicides evaluation, the isolates were grouped on the basis of growth rates on Petri plates with $90 \mathrm{~mm}$ diameter as fast (5-6 days), medium (8-10 days) and slow growing isolates (12-15 days) respectively. Among systemic fungicides, difenconazole and hexaconazole have completely inhibited growth of all the isolates of the S. rolfsii. While carbendazim was less effective in growth inhibition of $S$. rolfsii at all tested concentrations. Among non-systemic fungicides, mancozeb was more effective than chlorothalonil. All the fungicides tested effectively inhibited the growth of $S$. rolfsii isolates. Among these fungicides, hexaconazole and difenconazole (systemic) were superior at $125 \mathrm{ppm}$ and proved the most effective for suppressing the growth of $S$. rolfsii isolates followed by mancozeb and chlorothalonil (non-systemic).

\section{Introduction}

Sclerotium rolfsii Sacc. [teleomorph: Athelia rolfsii (Curzi) Tu \& Kimbrough, Corticiaceae, Basidiomycota] is a well-known ubiquitous soil inhabiting and most destructive soil borne fungus has been initially described by Rolfs (1892) on tomato. S. rolfsii, a soil borne plant pathogen of worldwide importance and has a very extensive host range of more than 500 plant species (Aycock, 1966). The fungus is distributed in both temperate and tropical regions (Punja, 1985; 1988; Gazaway and Hagan, 1989). The prolific growth rate of the fungus and an ability to produce large number of sclerotia that persist in the soil for several years, make the fungus a good facultative parasite with major importance as a pathogen throughout the world (Punja, 1988). The disease occurrence by $S$. rolfsii is typically endemic and the spatial distribution of the disease is clustered (Shew et al., 1984). The fungus spreads by mycelial contact with healthy plants and over-winters as sclerotia in 
soil. The sclerotia serve as the primary source of inoculum and are capable of initiating infection with or without an additional food base (Punja, 1985). Among the soil-borne diseases of groundnut, seed, stem and pod-rot caused by $S$. rolfsii is considered most economically important causing crop losses at different stages of crop growth in India (especially in Saurashtra region of Gujarat). It also causes root rot and leaf blight in groundnut (Amma and Shanmugam, 1976; Desai and Ghewande, 1991). The yield loss up to 27 per cent in groundnut due to this disease was also reported from India (Chohan, 1974). Mayee and Datar (1988) reported 25 per cent yield losses due to $S$. rolfsii in groundnut from Maharashtra. Field isolates of S. rolfsii from various host and geographical areas differed in growth rate, numbers and size of sclerotia produced (Punja and Grogan, 1983). Now days, fungicides are known to be the most effective method of disease control. Chemical control strategies remain the major tool in the management of stem rot of groundnut. Johnson et al., (2008) reported the fungicides hexaconazole, propiconazole, mancozeb completely inhibited the growth of S. rolfsii. Keeping the above in view the present work was taken up to evaluate some systemic and non-systemic fungicides against the pathogen $S$. rolfsii in vitro. The main objective of present study is to find out the effective fungicides to manage the yield losses of Groundnut due to Stem rot pathogen.

\section{Materials and Methods}

\section{Collection and maintenance of isolates}

The experiment was conducted at Department of Plant Pathology, College of Agriculture, Junagadh Agricultural University in the year 2007. Twenty isolates of Sclerotium rolfsii Sacc., from different hosts were collected from Indian Type Culture Collection (ITCC), IARI, New Delhi; National Research Centre for Groundnut (NRCG), Junagadh; Department of Plant Pathology, College of Agriculture, Junagadh Agricultural University, Junagadh and Department of Plant Pathology, Maharana Pratap University of Agriculture and Technology, Udaipur (Table 1) for in vitro evaluation of some fungicides (systemic and non-systemic) and were used in the present studies. Pure culture of isolates of S. rolfsii was maintained on potato dextrose agar medium aseptically for further studies.

\section{Evaluation of fungicides in vitro Condition}

For fungicide evaluation, the isolates were grouped on the basis of growth rates on petriplates with $90 \mathrm{~mm}$ diameter as fast (5-6 days), medium (8-10 days) and slow growing isolates (12-15 days) respectively. Different fungicides were tested for the effect on growth of $S$. rolfsii using poisoned-food technique (Dhingra and Sinclair, 1993).

In all experiments, potato dextrose agar medium (PDA) was used as basal medium. The required quantity of each chemical was incorporated aseptically in $100 \mathrm{ml}$ of autoclaved lukewarm PDA in $250 \mathrm{ml}$ conical flasks and was shaken well to facilitate uniform dispersal of the chemical and then immediately $20 \mathrm{ml}$ of medium was poured into each sterilized Petri plates and allowed to solidify.

The Petri plates were inoculated with $6 \mathrm{~mm}$ diameter mycelial discs, cut from the periphery of 10 days-old fungus cultures with the help of sterilized cork borer. The mycelial disc was placed in the centre of the plates in an inverted position to make a direct contact of mycelium with the poisoned medium and incubated at $26 \pm 1{ }^{\circ} \mathrm{C}$ for 5 to 15 days after inoculation. Simultaneously, a control was also maintained for each strain by growing the fungus on chemical-free PDA. The whole experiment was kept in triplicate (three 
replications) manner. Observation on linear growth was recorded only once when full growth of fungus was observed in control Petri plates.

Fungicides (Table 2) were tested using poisoned food technique. For systemic fungicides, the concentrations used were 0 , 125,250 and $500 \mathrm{ppm}$. For Non systemic ones, the concentrations were $0,500,1000$ and 2000 ppm.

Observations were recorded on linear growth and the per cent inhibition of growth of the fungus in each treatment was calculated by using following formula (Vincent, 1947).

$I=\frac{\mathrm{C}-\mathrm{T}}{\mathrm{C}}$

Where,

$\mathrm{I}=$ Per cent inhibition,

$\mathrm{C}=$ Colony diameter in control $(\mathrm{mm})$,

$\mathrm{T}=$ Colony diameter in respective treatment (mm)

\section{Statistical analysis of data}

Data were analyzed statistically to check the significance in variation and variability between different treatments and isolates of $S$. rolfsii using Factorial Completely Randomized Design (F-CRD) as following text (Rangaswami, 2002).

\section{Results and Discussion}

Twenty isolates of Sclerotium rolfsii Sacc. collected from different hosts and locations of India were studied in relation to efficacy of fungicides in vitro.

The results obtained in the present investigation are narrated herein.
Evaluation of fungicides against different isolates of Sclerotium rolfsii

In the present investigations five fungicides belonging to different groups viz., carbendazim, hexaconazole and difenoconazole from systemic; mancozeb and chlorothalonil from non-systemic groups were utilized. These fungicides were used to determine their relative efficacy against various isolates of Sclerotium rolfsii. The fungicides were evaluated by poisoned food technique and per cent growth inhibition was recorded at ranging from 125, 250 and 500 ppm for systemic; and 500, 1000 and 2000 ppm for non-systemic, respectively.

Effect of systemic fungicides on fast growing isolates of Sclerotium rolfsii

The efficacy of various systemic fungicides in inhibiting the growth of fast growing isolates of $S$. rolfsii were tested by using poisoned food technique at 125, 250 and $500 \mathrm{ppm}$ concentrations. Observations regarding per cent inhibition of growth recorded for various fungicidal concentrations are presented in Table 3, 4, 5 and depicted in Plate 1.

It is revealed from Table 3 that all main effects viz., Isolate (I), fungicide (F) and dose (D) as well as their interactions viz., I x F, I x D, F x D and I x F x D were found significant at five per cent level of significance. The minimum mean inhibition was 49.93 per cent for isolate M 1999, which was significantly lower than remaining eight isolates. Next in order was J 2004 II followed by J 2004 I, I 5543, I 5518, I 4743, I 4877 and I 5061; whereas, I $4630(86.22 \%)$ had shown the maximum mean inhibition. The lowest mean inhibition was observed for carbendazim (8.75 $\%)$. However, remaining two fungicides hexaconazole and difenoconazole had shown comparatively more inhibition i.e. 85.20 and 88.32 per cent, respectively. The increase in 
the concentration of doses significantly increased the inhibition per cent.

There was no inhibition recorded for carbendazim barring I 4630 and I 5543 isolates; whereas, cent per cent inhibition was recorded for I 4630 and I 5061 with hexaconazole and difenoconazole. Isolate I 4877 also showed cent per cent inhibition with difenoconazole. The interaction between I x D showed that inhibition was increased with increased in dose of fungicides (Table 4). It is also revealed from higher order interactions (I $\mathrm{x}$ D x F) given in Table 5 that isolates growth inhibition was not observed for carbendazim at all the concentration tested barring I 4630 and I 5543; whereas, cent per cent inhibition was recorded in I 4630 and I 5061 with their all three respective doses for hexaconazole and difenoconazole. A feathery growth was also showed in case of isolates I 5518 and J $2004 \mathrm{I}$ at $125 \mathrm{ppm}$ concentration with difenoconazole (Plate 1). Likewise, isolates $\mathbf{J}$ 2004 I, J 2004 II and M 1999 also had featheriness with difenoconazole at 250 and $500 \mathrm{ppm}$.

Effect of non-systemic fungicides on fast growing isolates of Sclerotium rolfsii

For testing the effect of non-systemic fungicides on fast growing isolates of $S$. rolfsii in vitro poisoned food technique was employed. The non-systemic fungicides were tested at 500, 1000 and $2000 \mathrm{ppm}$ concentrations.

The observations on the growth of the fungus in each treatment including check were recorded and calculated on the basis of the difference in growth obtained in the treatments and the check. Data regarding per cent inhibition of isolates growth are presented in Table 6, 7 and 8 . The comparative colony diameters of each treatment are depicted in Plate 2.
Data presented in Table 6 revealed that all main effects as well as the interaction effects were found significant at five per cent level of significance. The isolates J 2004 I, J 2004 II and I 5518 showed cent per cent mean inhibition. The lowest mean inhibition was found for I $5543(51.06 \%)$ followed by I 4877, M 1999, I 5061, I4630 and I 4743. Fungicide mancozeb was significantly lower than the chlorothalonil in the rate of inhibition. It was also observed that the inhibition per cent significantly increased with the increased in the concentration of the fungicide. The interaction of I $\mathrm{x} \mathrm{F}$ revealed that isolates I 5518, J 2004 I and J 2004 II showed cent per cent inhibition in case of both the fungicides (mancozeb and chlorothalonil).

Similar trend was also noticed in case of I x D interaction with all doses tested (Table 7). Isolates I 4630, I 4877, I 5061 and M 1999 (except I 4743) indicated significantly lower inhibition for mancozeb as compared to chlorothalonil. The higher order interaction (Table 8) of I x D x F showed that per cent growth inhibition was increased with increased in concentration of fungicides. However, the increased was much more from 500 to $1000 \mathrm{ppm}$ in case of chlorothalonil; whereas, it was marginal from 1000 to 2000 ppm for same fungicide when compared to mancozeb. It was also noticed that significantly lower inhibition was found at 500 ppm for chlorothalonil and mancozeb for isolates I $4877(21.33 \%)$ and I $5543(29 \%)$, respectively.

\section{Effect of systemic fungicides on medium growing isolates of Sclerotium rolfsii}

The relative efficacy of systemic fungicides inhibiting the growth of medium growing isolates of $S$. rolfsii was evaluated by poisoned food technique. The isolates were grown in Petri plates containing $20 \mathrm{ml}$ PDA supplemented with systemic fungicides at 
various concentrations as mentioned earlier. The data regarding per cent inhibition of the growth of isolates are presented in Table 9, 10 and 11 . The comparative colony diameter is depicted in Plate 3.

The mean inhibition per cent due to various treatments is given in Table 9. It is revealed from the Table 9 that all main and interaction effects were observed significant at five per cent level of significance. All isolates differed significantly and the lowest mean inhibition was observed in isolate I 5068 (66.67 \%). The maximum mean inhibition was observed in isolates I $4679(77.67 \%)$ followed by I 4725 (74.52\%), I $4724(71.85 \%)$ and finally the mean inhibition per cent were same $(73.00 \%)$ in I 4737 and I 5226 isolates. Out of three, two fungicides (hexaconazole and difenoconazole) have indicated cent per cent inhibition in all the six isolates. The lowest mean inhibition was observed for carbendazim $(18.35 \%)$. The inhibition was not seen in case of isolate I 5068 with fungicide carbendazim. Over all, per cent inhibition was increased with increased in concentration of fungicides for all isolates, except isolate I 5068 (Table 10). Data given for higher order interaction in Table 11 indicates the rate of per cent inhibition for carbendazim, hexaconazole and difenoconazole at different concentrations. No inhibition was observed for isolate I 5068 at lower to higher concentrations with carbendazim; whereas, cent per cent inhibition was found at lower to higher concentrations in all the six isolates with hexaconazole and difenoconazole. It was observed increased in concentration of carbendazim increased the per cent inhibition.

Effect of non-systemic fungicides on medium growing isolates of Sclerotium rolfsii

To study the relative efficacy of non-systemic fungicides on medium growing isolates of $S$. rolfsii in vitro; poisoned food technique was employed. The observations on the growth of the isolates were taken. The per cent inhibition was calculated on the basis of differences in growth observed in the treatments and check. Data regarding per cent growth inhibition of fungal isolates at various concentrations of fungicides are presented in Table 12, 13, 14 and depicted in Plate 4.

It is evident from the data presented in Table 12 that all main effects as well as the interaction effects were found significant at five per cent level of significance. The minimum per cent inhibition was found in isolate I 4679 (22.83\%); whereas, the cent per cent inhibition was observed in isolate I 4724 and I 5226. Isolates I 4724, I 4737 and I 5226 had cent per cent inhibition with mancozeb. In case of chlorothalonil, isolates I 4724 and I 5226 had cent per cent inhibition.

The increase in the concentration of fungicides increased the mean inhibition. Fungicide mancozeb was significantly higher than the chlorothalonil in inhibition. The interaction of I $x$ D revealed that all the isolates showed cent per cent inhibition in case of both the fungicides with D3 barring I 4679 and I 4725 under trial (Table 13).

Data pertaining to the higher order interactions given in Table 14 indicated increase in per cent inhibition with increased in concentrations of fungicides. The mancozeb and chlorothalonil inhibited cent per cent growth of isolates I 4724 and I 5226.

However, the growth inhibition was not noticed in isolate I 4679 for all concentrations of chlorothalonil tested. Similarly, isolate I 5068 exhibited nil growth at 500 ppm of chlorothalonil. Minimum growth inhibition 2.0 per cent was noticed in isolate I 5068 closely followed by isolate I 4679 at 500 ppm of mancozeb. 
Table.1 Collection of different isolates of Sclerotium rolfsii

\begin{tabular}{|c|c|c|c|c|}
\hline $\begin{array}{l}\text { Sr. } \\
\text { No. }\end{array}$ & $\begin{array}{c}\text { Isolates* } \\
\text { No. }\end{array}$ & Host & Common Name & Location and State \\
\hline 1. & I 4630 & - & Straw & Raipur (Chattisgarh) \\
\hline 2. & I 4679 & Plantago ovata Forsk. & Blonde psyllum & Bhubneshwar (Orissa) \\
\hline 3. & I 4723 & Tagetes minuta $\mathrm{L}$. & - & Nainital (Uttaranchal) \\
\hline 4. & I 4724 & Solanum tuberosum L. & Potato & Nainital (Uttaranchal) \\
\hline 5. & I 4725 & Canavalia gladiata (Jacq.) DC. & Sword bean & Nainital (Uttaranchal) \\
\hline 6. & I 4737 & $\begin{array}{l}\text { Catharanthus roseus (L.) G. } \\
\text { Don. }\end{array}$ & Periwinkle & New Delhi \\
\hline 7. & I 4743 & Dioscorea alata $\mathrm{L}$. & White yam & New Delhi \\
\hline 8. & I 4877 & Allium сера $\mathrm{L}$. & Onion & Dharwad (Maharashtra) \\
\hline 9. & I 4972 & Tagetes sp. & Marigold & Navasari (Gujarat) \\
\hline 10. & I 5061 & Unknown & - & IARI, New Delhi \\
\hline 11. & I 5068 & Lagerstroemia sp. & t & Hyderabad (Andhra Pradesh) \\
\hline 12. & I 5146 & Vigna unguiculata (L.) Walp. & Cowpea & Unknown \\
\hline 13. & I 5220 & Eleusine coracana (L.) Gaertn. & Finger millet & $\begin{array}{l}\text { Vellayani (Kerala) } \\
\end{array}$ \\
\hline 14. & I 5226 & $\begin{array}{l}\text { Pogostemon cablin (Blanco) } \\
\text { Benth. }\end{array}$ & Patchouli & New Delhi \\
\hline 15. & I 5518 & Nicotiana sp. & Tobacco & Lucknow (U.P.) \\
\hline 16. & I 5543 & Unknown & - & Thiruvanantpuram (Kerala) \\
\hline 17. & I 2782 & Capsicum annum $\mathrm{L}$. & Chilli & Unknown \\
\hline 18. & J 2004 I & Arachis hypogea $\mathrm{L}$ & Groundnut & Junagadh (Gujarat) \\
\hline 19. & J 2004 II & Capsicum annum $\mathrm{L}$. & Chilli & Junagadh (Gujarat) \\
\hline 20. & M 1999 & Arachis hypogea $\mathrm{L}$ & Groundnut & Udaipur (Rajasthan) \\
\hline
\end{tabular}

*Source:

01-17: Indian Type Culture Collection, IARI, New Delhi

18: National Research Centre for Groundnut, ICAR, Junagadh

19: Dept. of Plant Pathology, College of Agril., JAU: Junagadh

20: Dept. of Plant Pathology, Maharana Pratap University of Agriculture \& Technology, Udaipur, Rajasthan.

Table.2 Fungicides tested for their efficacy against different isolates of Sclerotium rolfsii in vitro

\begin{tabular}{|c|c|c|c|}
\hline \multicolumn{4}{|c|}{ Systemic fungicides } \\
\hline 1. & Carbendazim & Methyl $1 H$-benzimidazol-2-yl carbamate & Bavistin (50 \% WP) \\
\hline 2. & Difenoconazole & $\begin{array}{l}\text { 1-[2-[2-chloro-4-(4-chlorophenoxy) phenyl]-4- } \\
\text { methyl-1,3-dioxolon-2-ylmethyl]-1H-1,2,4-triazole }\end{array}$ & Score (25 \% EC) \\
\hline 3. & Hexaconazole & $\begin{array}{l}\text { ( } R S) \text {-2-(2,4-dichlorophenyl)-1-(1H-1,2,4-triazol-1- } \\
\text { yl) hexan-2-ol }\end{array}$ & Contaf (5 \% EC) \\
\hline \multicolumn{4}{|c|}{ Non - systemic fungicides } \\
\hline 4. & Mancozeb & Manganese ethylenebis (dithiocarbamate) & Dithane M-45 (75 \% WP) \\
\hline 5. & Chlorothalonil & Tetrachloroisophthalonitrile & Kavach (75 \% WP) \\
\hline
\end{tabular}


Table.3 Effect of systemic fungicides on fast growing isolates of S. rolfsii

\begin{tabular}{|c|c|c|c|c|}
\hline \multirow[t]{3}{*}{ Fungicide $(F)$} & \multicolumn{3}{|c|}{ Mean Inhibition \% (I x F) } & \multirow{3}{*}{$\begin{array}{c}\text { Mean } \\
\text { Inhibition } \\
\% \text { (I) }\end{array}$} \\
\hline & \multicolumn{3}{|c|}{ Fungicide (F) } & \\
\hline & Carbendazim & Hexaconazole & Difenoconazole & \\
\hline I 4630 & 58.67 & 100.00 & 100.00 & 86.22 \\
\hline I 4743 & 0.00 & 85.89 & 90.00 & 58.63 \\
\hline I 4877 & 0.00 & 96.00 & 100.00 & 65.34 \\
\hline I 5061 & 0.00 & 100.00 & 100.00 & 66.67 \\
\hline I 5518 & 0.00 & 84.33 & 88.33 & 57.56 \\
\hline I 5543 & 20.11 & 75.44 & 74.56 & 56.70 \\
\hline J 2004 I & 0.00 & 80.56 & 83.11 & 54.56 \\
\hline J 2004 II & 0.00 & 72.56 & 81.11 & 51.22 \\
\hline M 1999 & 0.00 & 72.00 & 77.78 & 49.93 \\
\hline \multicolumn{5}{|l|}{ Dose (ppm)(D) } \\
\hline $\mathrm{D}_{1}(\mathbf{1 2 5})$ & 6.89 & 80.22 & 82.82 & 56.64 \\
\hline $\mathrm{D}_{2}(250)$ & 8.85 & 85.56 & 89.85 & 61.42 \\
\hline $\mathrm{D}_{3}(500)$ & 10.52 & 89.82 & 92.30 & 64.21 \\
\hline Mean (Fungicide) & 8.75 & 85.20 & 88.32 & \multirow[b]{2}{*}{ CV \% } \\
\hline Effect & \multicolumn{3}{|l|}{ S.Em. } & \\
\hline $\mathbf{F}$ & \multicolumn{3}{|c|}{0.34} & \multirow{7}{*}{$\operatorname{cr} \%$} \\
\hline $\mathbf{I}$ & \multicolumn{3}{|c|}{0.60} & \\
\hline$F * I$ & \multicolumn{3}{|c|}{1.03} & \\
\hline $\mathbf{D}$ & \multicolumn{3}{|c|}{0.34} & \\
\hline$F^{* * D}$ & \multicolumn{3}{|c|}{0.60} & \\
\hline$I * D$ & \multicolumn{3}{|c|}{0.34} & \\
\hline$F * I * D$ & \multicolumn{3}{|c|}{1.80} & \\
\hline
\end{tabular}

Table.4 Interaction effect (I x D) of fast growing isolates of S. rolfsii with doses of systemic fungicides

\begin{tabular}{|c|c|c|c|}
\hline \multirow{2}{*}{ Isolate(I) } & \multicolumn{3}{|c|}{ Mean inhibition \% (I x D) } \\
\hline & D1 & D2 & D3 \\
\hline I 4630 & 83.89 & 87.11 & 87.67 \\
\hline I 4743 & 54.67 & 58.44 & 62.78 \\
\hline I 4877 & 62.67 & 66.67 & 66.67 \\
\hline I 5061 & 66.67 & 66.67 & 66.67 \\
\hline I 5518 & 48.11 & 61.78 & 62.78 \\
\hline I 5543 & 50.22 & 56.11 & 63.78 \\
\hline J 2004 I & 49.22 & 56.00 & 58.44 \\
\hline J 2004 II & 47.44 & 52.33 & 53.89 \\
\hline M 1999 & 46.89 & 47.67 & 55.22 \\
\hline
\end{tabular}


Table.5 Interaction effects of fast growing isolates of $S$. rolfsii, doses and systemic fungicides $(\mathrm{I} \times \mathrm{D} \times \mathrm{F})$

\begin{tabular}{|c|c|c|c|c|}
\hline \multirow{3}{*}{$\begin{array}{l}\text { Isolate } \\
\text { (I) }\end{array}$} & \multirow{3}{*}{$\begin{array}{c}\text { Conc. (ppm) } \\
\text { (D) }\end{array}$} & \multicolumn{3}{|c|}{ Mean Inhibition \% (I x D x F) } \\
\hline & & \multicolumn{3}{|c|}{ Fungicide(F) } \\
\hline & & Carbendazim & Hexaconazole & Difenoconazole \\
\hline \multirow[t]{3}{*}{ I 4630} & 125 & 51.67 & 100.00 & 100.00 \\
\hline & 250 & 61.33 & 100.00 & 100.00 \\
\hline & 500 & 63.00 & 100.00 & 100.00 \\
\hline \multirow[t]{3}{*}{ I 4743} & 125 & 0.00 & 82.67 & 81.33 \\
\hline & 250 & 0.00 & 86.67 & 88.67 \\
\hline & 500 & 0.00 & 88.33 & 100.00 \\
\hline \multirow{3}{*}{ I 4877} & 125 & 0.00 & 88.00 & 100.00 \\
\hline & 250 & 0.00 & 100.00 & 100.00 \\
\hline & 500 & 0.00 & 100.00 & 100.00 \\
\hline \multirow[t]{3}{*}{ I 5061} & 125 & 0.00 & 100.00 & 100.00 \\
\hline & 250 & 0.00 & 100.00 & 100.00 \\
\hline & 500 & 0.00 & 100.00 & 100.00 \\
\hline \multirow[t]{3}{*}{ I 5518} & 125 & 0.00 & 79.33 & 65.00 \\
\hline & 250 & 0.00 & 85.33 & 100.00 \\
\hline & 500 & 0.00 & 88.33 & 100.00 \\
\hline \multirow[t]{3}{*}{ I 5543} & 125 & 10.33 & 70.67 & 69.67 \\
\hline & 250 & 18.33 & 74.67 & 75.33 \\
\hline & 500 & 31.67 & 81.00 & 78.67 \\
\hline \multirow[t]{3}{*}{ J 2004 I } & 125 & 0.00 & 70.33 & 77.33 \\
\hline & 250 & 0.00 & 83.67 & 84.33 \\
\hline & 500 & 0.00 & 87.67 & 87.67 \\
\hline \multirow[t]{3}{*}{ J 2004 II } & 125 & 0.00 & 67.67 & 74.67 \\
\hline & 250 & 0.00 & 74.67 & 82.33 \\
\hline & 500 & 0.00 & 75.33 & 86.33 \\
\hline \multirow[t]{3}{*}{ M 1999} & 125 & 0.00 & 63.33 & 77.33 \\
\hline & 250 & 0.00 & 65.00 & 78.00 \\
\hline & 500 & 0.00 & 87.67 & 78.00 \\
\hline
\end{tabular}


Table.6 Effect of non-systemic fungicides on fast growing isolates of $S$. rolfsii

\begin{tabular}{|c|c|c|c|}
\hline \multirow[t]{2}{*}{ Fungicide $(\mathrm{F})$} & \multicolumn{2}{|c|}{ Mean Inhibition \% (I x F) } & \multirow{3}{*}{$\begin{array}{c}\text { Mean } \\
\text { Inhibition \% } \\
\text { (I) }\end{array}$} \\
\hline & & e (F) & \\
\hline Isolate (I) & Mancozeb & Chlorothalonil & \\
\hline I 4630 & 71.89 & 100.00 & 85.94 \\
\hline I 4743 & 100.00 & 74.33 & 87.17 \\
\hline I 4877 & 65.67 & 73.78 & 69.72 \\
\hline I 5061 & 63.22 & 100.00 & 81.61 \\
\hline I 5518 & 100.00 & 100.00 & 100.00 \\
\hline I 5543 & 48.78 & 53.33 & 51.06 \\
\hline J 2004 I & 100.00 & 100.00 & 100.00 \\
\hline J 2004 II & 100.00 & 100.00 & 100.00 \\
\hline M 1999 & 61.56 & 83.00 & 72.28 \\
\hline \multicolumn{4}{|l|}{ Dose (ppm) } \\
\hline $\mathrm{D}_{1}(500)$ & 65.74 & 71.74 & 68.74 \\
\hline$D_{2}(1000)$ & 76.74 & 93.04 & 84.89 \\
\hline$D_{3}(2000)$ & 94.56 & 96.70 & 95.63 \\
\hline Mean (Fungicide) & 79.01 & 87.16 & \\
\hline Effect & S.Em. & C.D. & CV \% \\
\hline $\mathbf{F}$ & 0.14 & 0.38 & \multirow{7}{*}{1.46} \\
\hline I & 0.29 & 0.80 & \\
\hline$F^{*} \mathrm{I}$ & 0.41 & 1.14 & \\
\hline D & 0.17 & 0.46 & \\
\hline$F * D$ & 0.23 & 0.66 & \\
\hline I*D & 0.50 & 0.46 & \\
\hline$F * I * D$ & 0.70 & 1.97 & \\
\hline
\end{tabular}

Table.7 Interaction effect (I x D) of fast growing isolates of S. rolfsii with doses of non-systemic fungicides

\begin{tabular}{|c|c|c|c|}
\hline Isolate & \multicolumn{3}{|c|}{ Dose(D) } \\
\hline & D1 & D2 & D3 \\
\hline I 4630 & 71.00 & 92.67 & 94.17 \\
\hline I 4743 & 71.83 & 89.67 & 100.00 \\
\hline I 4877 & 32.17 & 77.00 & 100.00 \\
\hline I 5061 & 70.33 & 74.50 & 100.00 \\
\hline I 5518 & 100.00 & 100.00 & 100.00 \\
\hline I 5543 & 30.33 & 56.33 & 66.50 \\
\hline J 2004 I & 100.00 & 100.00 & 100.00 \\
\hline J 2004 II & 100.00 & 100.00 & 100.00 \\
\hline M 1999 & 43.00 & 73.83 & 100.00 \\
\hline
\end{tabular}


Table.8 Interaction effects of fast growing isolates of $S$. rolfsii, doses and non-systemic fungicides (I x D x F)

\begin{tabular}{|c|c|c|c|}
\hline \multirow{3}{*}{$\begin{array}{l}\text { Isolate } \\
\text { (I) }\end{array}$} & \multirow{3}{*}{$\begin{array}{l}\text { Conc. (ppm) } \\
\text { (D) }\end{array}$} & \multicolumn{2}{|c|}{ Mean Inhibition \% (I x D x F) } \\
\hline & & \multicolumn{2}{|c|}{ Fungicide (F) } \\
\hline & & Mancozeb & Chlorothalonil \\
\hline \multirow[t]{3}{*}{ I 4630} & 500 & 42.00 & 100.00 \\
\hline & 1000 & 85.33 & 100.00 \\
\hline & 2000 & 88.33 & 100.00 \\
\hline \multirow{3}{*}{ I 4743} & 500 & 100.00 & 43.67 \\
\hline & 1000 & 100.00 & 79.33 \\
\hline & 2000 & 100.00 & 100.00 \\
\hline \multirow[t]{3}{*}{ I 4877} & 500 & 43.00 & 21.33 \\
\hline & 1000 & 54.00 & 100.00 \\
\hline & 2000 & 100.00 & 100.00 \\
\hline \multirow[t]{3}{*}{ I 5061} & 500 & 40.67 & 100.00 \\
\hline & 1000 & 49.00 & 100.00 \\
\hline & 2000 & 100.00 & 100.00 \\
\hline \multirow[t]{3}{*}{ I 5518} & 500 & 100.00 & 100.00 \\
\hline & 1000 & 100.00 & 100.00 \\
\hline & 2000 & 100.00 & 100.00 \\
\hline \multirow[t]{3}{*}{ I 5543} & 500 & 29.00 & 31.67 \\
\hline & 1000 & 54.67 & 58.00 \\
\hline & 2000 & 62.67 & 70.33 \\
\hline \multirow[t]{3}{*}{$\mathrm{J} 2004 \mathrm{I}$} & 500 & 100.00 & 100.00 \\
\hline & 1000 & 100.00 & 100.00 \\
\hline & 2000 & 100.00 & 100.00 \\
\hline \multirow[t]{3}{*}{ J 2004 II } & 500 & 100.00 & 100.00 \\
\hline & 1000 & 100.00 & 100.00 \\
\hline & 2000 & 100.00 & 100.00 \\
\hline \multirow[t]{3}{*}{ M 1999} & 500 & 37.00 & 49.00 \\
\hline & 1000 & 47.67 & 100.00 \\
\hline & 2000 & 100.00 & 100.00 \\
\hline
\end{tabular}


Table.9 Effect of systemic fungicides on medium growing isolates of $S$. rolfsii

\begin{tabular}{|c|c|c|c|c|}
\hline \multirow{3}{*}{$\begin{array}{l}\text { Fungicide (F) } \\
\text { Isolate (I) }\end{array}$} & \multicolumn{3}{|c|}{ Mean Inhibition \% (I x F) } & \multirow{3}{*}{$\begin{array}{c}\text { Mean } \\
\text { Inhibition } \\
\% \text { (I) }\end{array}$} \\
\hline & \multicolumn{3}{|c|}{ Fungicide (F) } & \\
\hline & Carbendazim & Hexaconazole & Difenoconazole & \\
\hline I 4679 & 33.00 & 100.00 & 100.00 & 77.67 \\
\hline I 4724 & 15.56 & 100.00 & 100.00 & 71.85 \\
\hline I 4725 & 23.56 & 100.00 & 100.00 & 74.52 \\
\hline I 4737 & 19.00 & 100.00 & 100.00 & 73.00 \\
\hline I 5068 & 0.00 & 100.00 & 100.00 & 66.67 \\
\hline I 5226 & 19.00 & 100.00 & 100.00 & 73.00 \\
\hline \multicolumn{5}{|l|}{ Dose (ppm) } \\
\hline $\mathrm{D}_{1}(\mathbf{1 2 5})$ & 9.94 & 100.00 & 100.00 & 69.98 \\
\hline $\mathrm{D}_{2}(\mathbf{2 5 0})$ & 14.94 & 100.00 & 100.00 & 71.65 \\
\hline $\mathrm{D}_{3}(500)$ & 30.17 & 100.00 & 100.00 & 76.72 \\
\hline $\begin{array}{c}\text { Mean } \\
\text { (Fungicide) }\end{array}$ & 18.35 & 100.00 & 100.00 & \\
\hline Effect & \multicolumn{3}{|l|}{ S.Em. } & CV\% \\
\hline $\mathbf{F}$ & 0.14 & \multicolumn{2}{|c|}{0.38} & \multirow{7}{*}{1.38} \\
\hline $\mathbf{I}$ & 0.19 & \multicolumn{2}{|c|}{0.54} & \\
\hline$F * I$ & 0.33 & \multicolumn{2}{|c|}{0.94} & \\
\hline$\overline{\mathbf{D}}$ & 0.14 & \multicolumn{2}{|c|}{0.38} & \\
\hline F*D & 0.24 & \multicolumn{2}{|c|}{0.66} & \\
\hline $\mathbf{I} * \mathbf{D}$ & 0.33 & \multicolumn{2}{|c|}{0.38} & \\
\hline$F * I * D$ & 0.58 & \multicolumn{2}{|c|}{1.63} & \\
\hline
\end{tabular}

Table.10 Interaction effect (I x D) of medium growing isolates of $S$. rolfsii with doses of systemic fungicides

\begin{tabular}{|c|c|c|c|}
\hline $\begin{array}{c}\text { Isolate } \\
\text { (I) }\end{array}$ & D1 & Dose(D) & D3 \\
\hline I 4679 & 74.00 & 79.11 & 79.89 \\
\hline I 4724 & 71.11 & 71.56 & 72.89 \\
\hline I 4725 & 71.89 & 75.33 & 76.33 \\
\hline I 4737 & 68.67 & 69.22 & 81.11 \\
\hline I 5068 & 66.67 & 66.67 & 66.67 \\
\hline I 5226 & 67.56 & 68.00 & 83.44 \\
\hline
\end{tabular}


Table.11 Interaction effects of medium growing isolates of $S$. rolfsii, doses and systemic fungicides ( $\mathrm{x}$ D x F)

\begin{tabular}{|c|c|c|c|c|}
\hline \multirow{3}{*}{$\begin{array}{l}\text { Isolate } \\
\text { (I) }\end{array}$} & \multirow{3}{*}{$\begin{array}{l}\text { Conc. (ppm) } \\
\text { (D) }\end{array}$} & \multicolumn{3}{|c|}{ Mean Inhibition \% (I x D x F) } \\
\hline & & \multicolumn{3}{|c|}{ Fungicide (F) } \\
\hline & & Carbendazim & Hexaconazole & Difenoconazole \\
\hline \multirow[t]{3}{*}{ I 4679} & 125 & 22.00 & 100.00 & 100.00 \\
\hline & 250 & 37.33 & 100.00 & 100.00 \\
\hline & 500 & 39.67 & 100.00 & 100.00 \\
\hline \multirow[t]{3}{*}{ I 4724} & 125 & 13.33 & 100.00 & 100.00 \\
\hline & 250 & 14.67 & 100.00 & 100.00 \\
\hline & 500 & 18.67 & 100.00 & 100.00 \\
\hline \multirow[t]{3}{*}{ I 4725} & 125 & 15.67 & 100.00 & 100.00 \\
\hline & 250 & 26.00 & 100.00 & 100.00 \\
\hline & 500 & 29.00 & 100.00 & 100.00 \\
\hline \multirow[t]{3}{*}{ I 4737} & 125 & 6.00 & 100.00 & 100.00 \\
\hline & 250 & 7.67 & 100.00 & 100.00 \\
\hline & 500 & 43.33 & 100.00 & 100.00 \\
\hline \multirow[t]{3}{*}{ I 5068} & 125 & 0.00 & 100.00 & 100.00 \\
\hline & 250 & 0.00 & 100.00 & 100.00 \\
\hline & 500 & 0.00 & 100.00 & 100.00 \\
\hline \multirow[t]{3}{*}{ I 5226} & 125 & 2.67 & 100.00 & 100.00 \\
\hline & 250 & 4.00 & 100.00 & 100.00 \\
\hline & 500 & 50.33 & 100.00 & 100.00 \\
\hline
\end{tabular}

Table.12 Effect of non-systemic fungicides on medium growing isolates of $S$. rolfsii

\begin{tabular}{|c|c|c|c|}
\hline \multirow{3}{*}{$\begin{array}{l}\text { Fungicide (F) } \\
\text { Isolate (I) }\end{array}$} & \multicolumn{2}{|c|}{ Mean Inhibition \% (I x F) } & \multirow{3}{*}{$\begin{array}{c}\text { Mean } \\
\text { Inhibition \% (I) }\end{array}$} \\
\hline & \multicolumn{2}{|c|}{ Fungicide (F) } & \\
\hline & Mancozeb & Chlorothalonil & \\
\hline I 4679 & 45.67 & 0.00 & 22.83 \\
\hline I 4724 & 100.00 & 100.00 & 100.00 \\
\hline I 4725 & 50.44 & 24.00 & 37.22 \\
\hline I 4737 & 100.00 & 47.22 & 73.61 \\
\hline I 5068 & 37.44 & 66.67 & 52.06 \\
\hline I5226 & 100.00 & 100.00 & 100.00 \\
\hline \multicolumn{4}{|l|}{ Dose (ppm) } \\
\hline$D_{1}(500)$ & 58.11 & 40.39 & 49.25 \\
\hline$D_{2}(1000)$ & 70.22 & 57.67 & 63.94 \\
\hline $\mathrm{D}_{3}(2000)$ & 88.44 & 70.89 & 79.67 \\
\hline Mean (Fungicide) & 72.26 & 56.32 & \\
\hline Effect & S.Em. & C.D. & CV \% \\
\hline $\mathbf{F}$ & 0.15 & 0.43 & \multirow{7}{*}{1.75} \\
\hline $\mathbf{I}$ & 0.27 & 0.75 & \\
\hline$F * I$ & 0.38 & 1.06 & \\
\hline $\bar{D}$ & 0.19 & 0.53 & \\
\hline$F * D$ & 0.27 & 0.75 & \\
\hline I*D & 0.46 & 0.53 & \\
\hline$F * I * D$ & 0.65 & 1.83 & \\
\hline
\end{tabular}


Table.13 Interaction effect (I x D) of medium growing isolates of $S$. rolfsii with doses of nonsystemic fungicides

\begin{tabular}{|c|c|c|c|}
\hline $\begin{array}{c}\text { Isolate } \\
\text { (I) }\end{array}$ & D1 & Dose (D) & D2 \\
\hline I 4679 & 2.00 & 30.00 & 36.50 \\
\hline I 4724 & 100.00 & 100.00 & 100.00 \\
\hline I 4725 & 33.00 & 37.17 & 41.50 \\
\hline I 4737 & 59.50 & 61.33 & 100.00 \\
\hline I5068 & 1.00 & 55.17 & 100.00 \\
\hline I5226 & 100.00 & 100.00 & 100.00 \\
\hline
\end{tabular}

Table.14 Interaction effects of medium growing isolates of $S$. rolfsii, doses and non-systemic fungicides ( $\mathrm{I} \times \mathrm{D} \times \mathrm{F})$

\begin{tabular}{|c|c|c|c|}
\hline \multirow{3}{*}{$\begin{array}{l}\text { Isolate } \\
\text { (I) }\end{array}$} & \multirow{3}{*}{$\begin{array}{l}\text { Conc. (ppm) } \\
\text { (D) }\end{array}$} & \multicolumn{2}{|c|}{ Mean Inhibition \% (I x D x F ) } \\
\hline & & \multicolumn{2}{|c|}{ Fungicide (F) } \\
\hline & & Mancozeb & Chlorothalonil \\
\hline \multirow[t]{3}{*}{$\bar{I} 4679$} & 500 & 4.00 & 0.00 \\
\hline & 1000 & 60.00 & 0.00 \\
\hline & 2000 & 73.00 & 0.00 \\
\hline \multirow[t]{3}{*}{ I 4724} & 500 & 100.00 & 100.00 \\
\hline & 1000 & 100.00 & 100.00 \\
\hline & 2000 & 100.00 & 100.00 \\
\hline \multirow[t]{3}{*}{ I 4725} & 500 & 42.67 & 23.33 \\
\hline & 1000 & 51.00 & 23.33 \\
\hline & 2000 & 57.67 & 25.33 \\
\hline \multirow[t]{3}{*}{ I 4737} & 500 & 100.00 & 19.00 \\
\hline & 1000 & 100.00 & 22.67 \\
\hline & 2000 & 100.00 & 100.00 \\
\hline \multirow[t]{3}{*}{ I5068 } & 500 & 2.00 & 0.00 \\
\hline & 1000 & 10.33 & 100.00 \\
\hline & 2000 & 100.00 & 100.00 \\
\hline \multirow[t]{3}{*}{$\overline{I 5226}$} & 500 & 100.00 & 100.00 \\
\hline & 1000 & 100.00 & 100.00 \\
\hline & 2000 & 100.00 & 100.00 \\
\hline
\end{tabular}


Table.15 Effect of systemic fungicides on slow growing isolates of S. rolfsii

\begin{tabular}{|c|c|c|c|c|}
\hline \multirow{3}{*}{$\begin{array}{l}\text { Fungicide (F) } \\
\text { Isolate (I) }\end{array}$} & \multicolumn{3}{|c|}{ Mean Inhibition \% (I x F) } & \multirow{3}{*}{$\begin{array}{c}\text { Mean } \\
\text { Inhibition } \\
\% \text { (I) }\end{array}$} \\
\hline & \multicolumn{3}{|c|}{ Fungicide (F) } & \\
\hline & Carbendazim & Hexaconazole & Difenoconazole & \\
\hline I 4723 & 91.00 & 91.67 & 77.11 & 86.59 \\
\hline I 4972 & 100.00 & 77.78 & 85.33 & 87.70 \\
\hline I 5146 & 15.44 & 100.00 & 89.22 & 68.22 \\
\hline I 5220 & 0.00 & 100.00 & 100.00 & 66.67 \\
\hline I 2782 & 87.44 & 100.00 & 100.00 & 95.82 \\
\hline \multicolumn{5}{|l|}{ Dose (ppm) } \\
\hline$D_{1}(125)$ & 47.07 & 89.53 & 87.93 & 74.84 \\
\hline $\mathrm{D}_{2}(250)$ & 61.00 & 95.00 & 89.00 & 81.67 \\
\hline $\mathbf{D}_{3}(500)$ & 68.26 & 97.13 & 94.07 & 86.49 \\
\hline Mean (Fungicide) & 58.78 & 93.89 & 90.33 & \\
\hline Effect & S.Em. & C.D. & \multicolumn{2}{|l|}{ CV \% } \\
\hline $\bar{F}$ & 0.15 & 0.42 & \multirow{7}{*}{1.24} & \\
\hline $\mathbf{I}$ & 0.19 & 0.55 & & \\
\hline$F * I$ & 0.34 & 0.95 & & \\
\hline$\overline{\mathbf{D}}$ & 0.15 & 0.42 & & \\
\hline$F * D$ & 0.26 & 0.73 & & \\
\hline$I * D$ & 0.34 & 0.42 & & \\
\hline$F^{*} I * D$ & 0.58 & 1.64 & & \\
\hline
\end{tabular}

Table.16 Interaction effect (I x D) of slow growing isolates of S. rolfsii with doses of systemic fungicides

\begin{tabular}{|c|rr|r|r|}
\hline Isolate & \multicolumn{4}{|c|}{ Dose (D) } \\
\hline & D1 & \multicolumn{1}{|c|}{ D2 } & \multicolumn{1}{c|}{ D3 } \\
\hline I4723 & & 73.89 & 91.67 & 94.22 \\
\hline I 4972 & & 85.11 & 86.89 & 91.11 \\
\hline I5146 & & 61.11 & 63.11 & 80.44 \\
\hline I5220 & & 66.67 & 66.67 & 66.67 \\
\hline I 2782 & & 87.44 & 100.00 & 100.00 \\
\hline
\end{tabular}


Table.17 Interaction effects of slow growing isolates of $S$. rolfsii, doses and systemic fungicides $(\mathrm{I} \times \mathrm{D} \times \mathrm{F})$

\begin{tabular}{|c|c|c|c|c|}
\hline \multirow{3}{*}{$\begin{array}{l}\text { Isolate } \\
\text { (I) }\end{array}$} & \multirow{3}{*}{$\begin{array}{c}\text { Conc. (ppm) } \\
\text { (D) }\end{array}$} & \multicolumn{3}{|c|}{ Mean Inhibition \% (I x D x F) } \\
\hline & & \multicolumn{3}{|c|}{ Fungicide(F) } \\
\hline & & Carbendazim & Hexaconazole & Difenoconazole \\
\hline \multirow[t]{3}{*}{ I 4723} & 125 & 73.00 & 75.00 & 73.67 \\
\hline & 250 & 100.00 & 100.00 & 75.00 \\
\hline & 500 & 100.00 & 100.00 & 82.67 \\
\hline \multirow[t]{3}{*}{ I 4972} & 125 & 100.00 & 72.67 & 82.67 \\
\hline & 250 & 100.00 & 75.00 & 85.67 \\
\hline & 500 & 100.00 & 85.67 & 87.67 \\
\hline \multirow{3}{*}{ I 5146} & 125 & 0.00 & 100.00 & 83.33 \\
\hline & 250 & 5.00 & 100.00 & 84.33 \\
\hline & 500 & 41.33 & 100.00 & 100.00 \\
\hline \multirow[t]{3}{*}{ I 5220} & 125 & 0.00 & 100.00 & 100.00 \\
\hline & 250 & 0.00 & 100.00 & 100.00 \\
\hline & 500 & 0.00 & 100.00 & 100.00 \\
\hline \multirow[t]{3}{*}{ I 2782} & 125 & 62.33 & 100.00 & 100.00 \\
\hline & 250 & 100.00 & 100.00 & 100.00 \\
\hline & 500 & 100.00 & 100.00 & 100.00 \\
\hline
\end{tabular}

Table.18 Effect of non-systemic fungicides on slow growing isolates of $S$. rolfsii

\begin{tabular}{|c|c|c|c|}
\hline \multirow{3}{*}{$\begin{array}{l}\text { Fungicide }(\mathrm{F}) \\
\text { Isolate (I) }\end{array}$} & \multicolumn{2}{|c|}{ Mean Inhibition \%(I x F) } & \multirow{3}{*}{$\begin{array}{c}\text { Mean } \\
\text { Inhibition \% } \\
\text { (I) }\end{array}$} \\
\hline & \multicolumn{2}{|c|}{ Fungicide (F) } & \\
\hline & Mancozeb & Chlorothalonil & \\
\hline I 4723 & 62.00 & 88.33 & 75.17 \\
\hline I 4972 & 68.00 & 84.67 & 76.33 \\
\hline I5146 & 100.00 & 66.67 & 83.33 \\
\hline $\mathbf{I 5 2 2 0}$ & 66.67 & 53.78 & 60.22 \\
\hline I 2782 & 41.22 & 46.78 & 44.00 \\
\hline \multicolumn{4}{|l|}{ Dose (ppm) } \\
\hline $\mathrm{D}_{1}(500)$ & 39.07 & 27.80 & 33.43 \\
\hline $\mathrm{D}_{2}(1000)$ & 63.67 & 76.33 & 70.00 \\
\hline $\mathrm{D}_{3}(2000)$ & 100.00 & 100.00 & 100.00 \\
\hline Mean (Fungicide) & 67.58 & 68.04 & \\
\hline Effect & S.Em. & C.D. & CV\% \\
\hline $\bar{F}$ & 0.20 & NS & \multirow{7}{*}{1.98} \\
\hline $\mathbf{I}$ & 0.32 & 0.89 & \\
\hline$F * I$ & 0.45 & 1.27 & \\
\hline $\mathbf{D}$ & 0.25 & 0.69 & \\
\hline $\mathbf{F} * \mathbf{D}$ & 0.35 & 0.98 & \\
\hline $\mathbf{I} * \mathbf{D}$ & 0.55 & 0.69 & \\
\hline$F * I * D$ & 0.78 & 2.19 & \\
\hline
\end{tabular}


Table.19 Interaction effect (I x D) of slow growing isolates of S. rolfsii with doses of nonsystemic fungicides

\begin{tabular}{|c|c|c|c|}
\hline $\begin{array}{c}\text { Isolate } \\
\text { (I) }\end{array}$ & \multicolumn{3}{|c|}{ Dose (D) } \\
\hline I 4723 & D1 & D2 & D3 \\
\hline I 4972 & 52.50 & 73.00 & 100.00 \\
\hline I 5146 & 52.17 & 76.83 & 100.00 \\
\hline I 5220 & 50.00 & 100.00 & 100.00 \\
\hline I 2782 & 6.17 & 74.50 & 100.00 \\
\hline
\end{tabular}

Table.20 Interaction effects of slow growing isolates of $S$. rolfsii, doses and non-systemic fungicides ( $\mathrm{x}$ D x F)

\begin{tabular}{|c|c|c|c|}
\hline \multirow{3}{*}{$\begin{array}{l}\text { Isolate } \\
\text { (I) }\end{array}$} & \multirow{3}{*}{$\begin{array}{c}\text { Conc. (ppm) } \\
\text { (D) }\end{array}$} & \multicolumn{2}{|c|}{ Mean Inhibition \% (I x D x F) } \\
\hline & & \multicolumn{2}{|c|}{ Fungicide(F) } \\
\hline & & Mancozeb & Chlorothalonil \\
\hline \multirow[t]{3}{*}{ I 4723} & 500 & 40.00 & 65.00 \\
\hline & 1000 & 46.00 & 100.00 \\
\hline & 2000 & 100.00 & 100.00 \\
\hline \multirow[t]{3}{*}{ I 4972} & 500 & 50.33 & 54.00 \\
\hline & 1000 & 53.67 & 100.00 \\
\hline & 2000 & 100.00 & 100.00 \\
\hline \multirow[t]{3}{*}{ I 5146 } & 500 & 100.00 & 0.00 \\
\hline & 1000 & 100.00 & 100.00 \\
\hline & 2000 & 100.00 & 100.00 \\
\hline \multirow[t]{3}{*}{ I 5220} & 500 & 0.00 & 12.33 \\
\hline & 1000 & 100.00 & 49.00 \\
\hline & 2000 & 100.00 & 100.00 \\
\hline \multirow[t]{3}{*}{ I 2782} & 500 & 5.00 & 7.67 \\
\hline & 1000 & 18.67 & 32.67 \\
\hline & 2000 & 100.00 & 100.00 \\
\hline
\end{tabular}




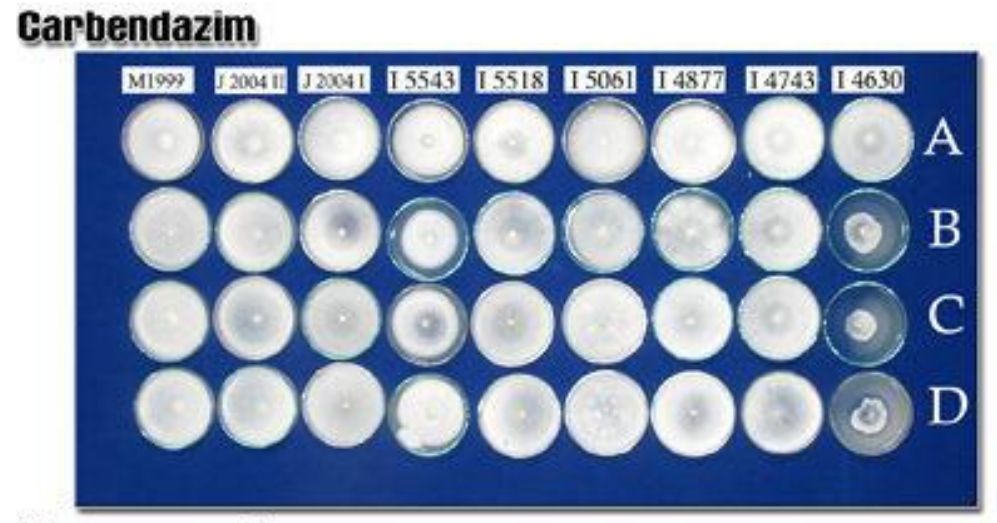

\section{Hexaconazole}

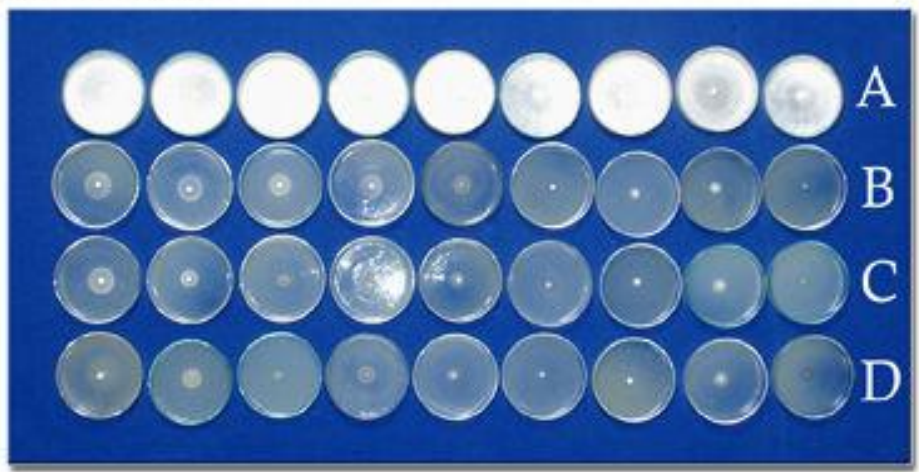

\section{Difenoconazole}

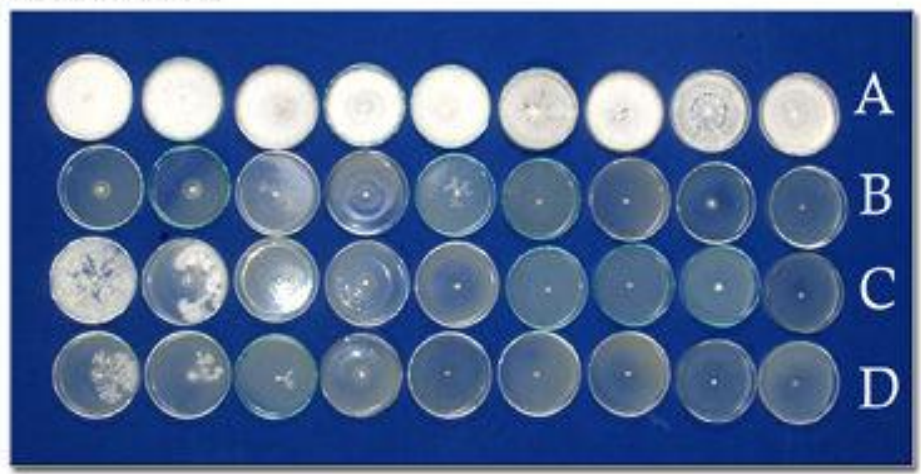

\section{Plate1: Effect of systemic fungicides on fast} growing isolates of Sclerotium rolfsii.

Concentrations : $\begin{array}{ll}A=0 \mathrm{ppm} & C=250 \mathrm{ppm} \\ B=125 \mathrm{ppm} & D=500 \mathrm{ppm}\end{array}$




\section{Mencozeb}

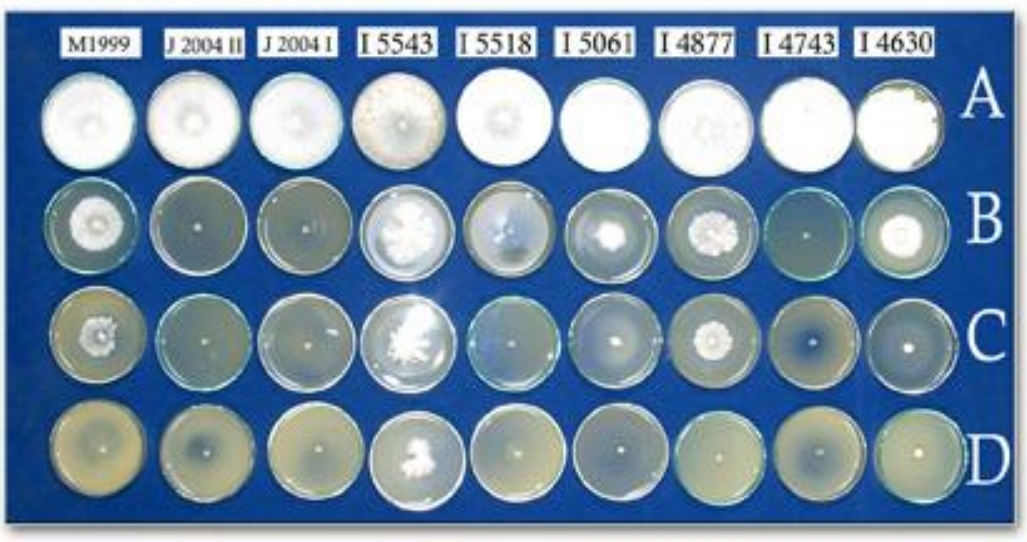

\section{Chlorothalonil}

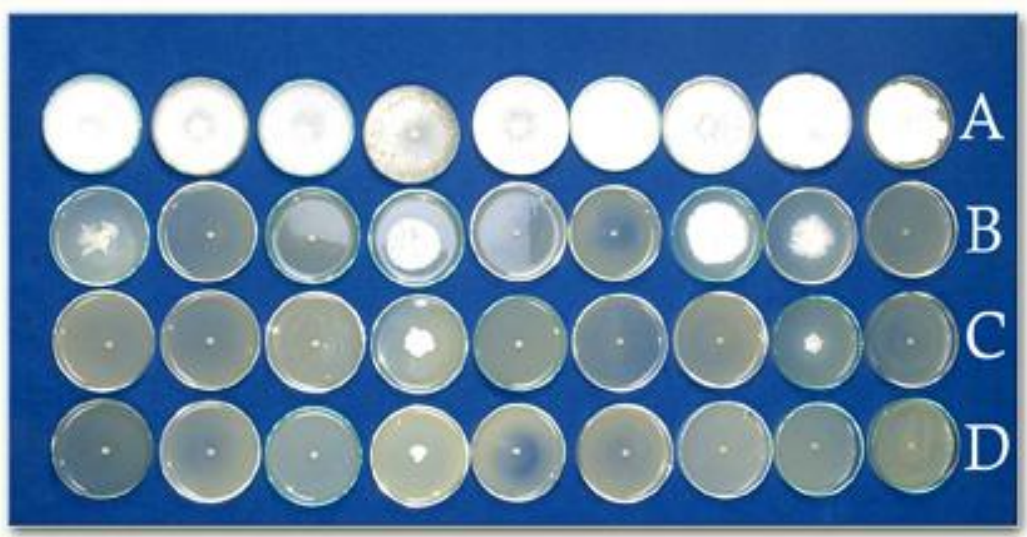

\section{Plate 2 ; Effect of non - systemic fungicides on} tast growing isolates of Sclerotium rolfsii.

$$
\text { Concentrations : } \begin{aligned}
A & =0 \mathrm{ppm} \\
\mathbf{B} & =500 \mathrm{ppm} \\
\mathbf{C} & =1000 \mathrm{pm} \\
\mathbf{D} & =2000 \mathrm{ppm}
\end{aligned}
$$




\section{Carbendazim}

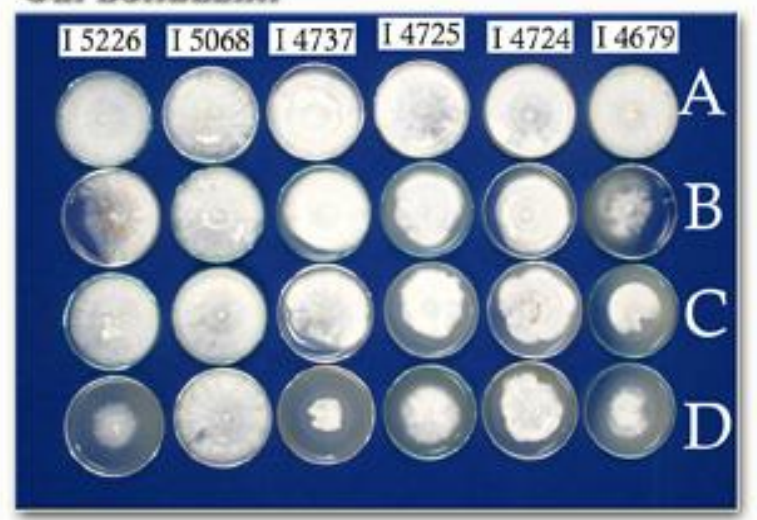

\section{Hexaconazole}

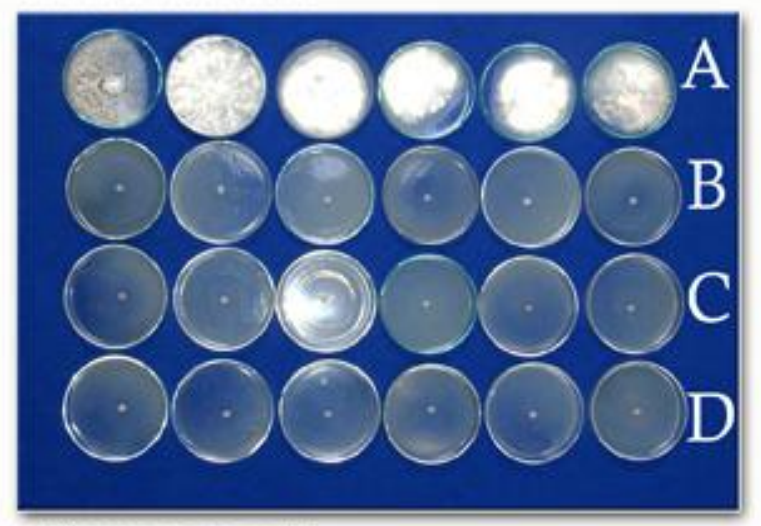

\section{Difenoconazole}

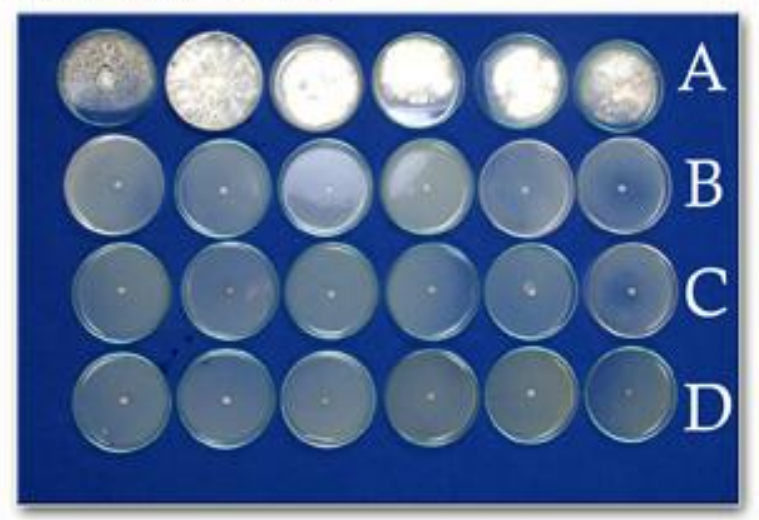

\section{Plate 3 : Effect of systemic fungicides on medium} growing isolates of Sclerotium rolfsii. 


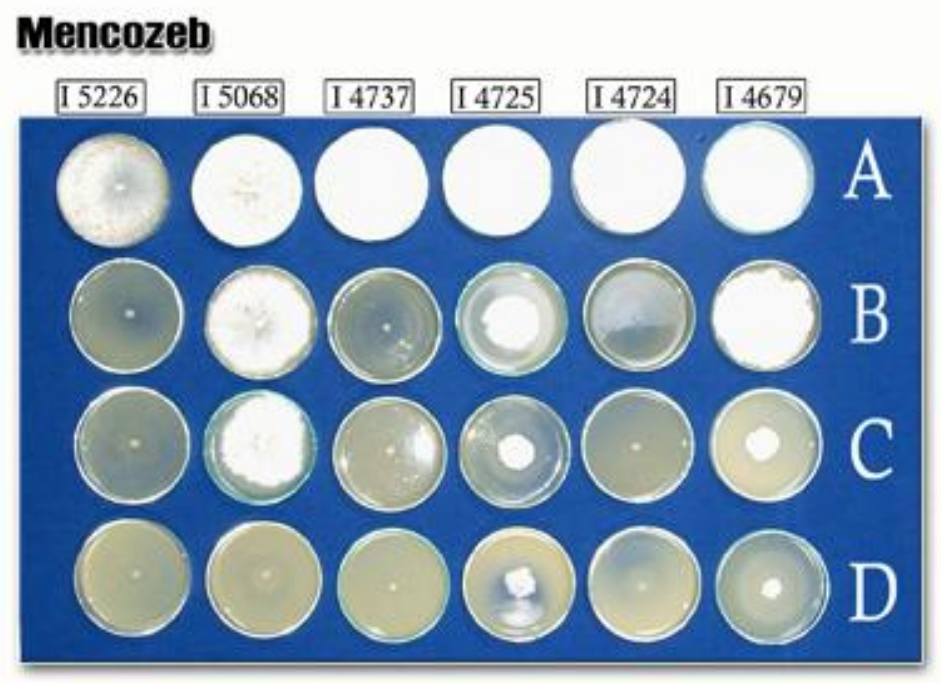

\section{Chlorothalonil}

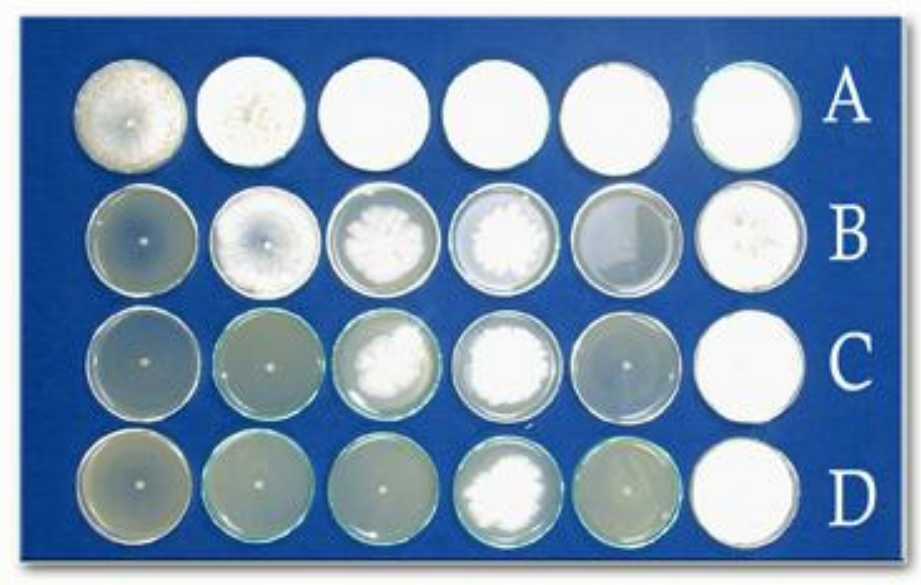

\section{Plate 4 : Effect of non - systemic fungicides on} medium growing isolates of Sclerotium rolfsii,

Concentrations : A - 0 ppm

$B=500 \mathrm{ppm}$

$\mathrm{C}=1000 \mathrm{p} \mathrm{Dm}$

$\mathrm{D}=\mathbf{2 0 0 0 \mathrm { ppm }}$ 


\section{Carbendazim}

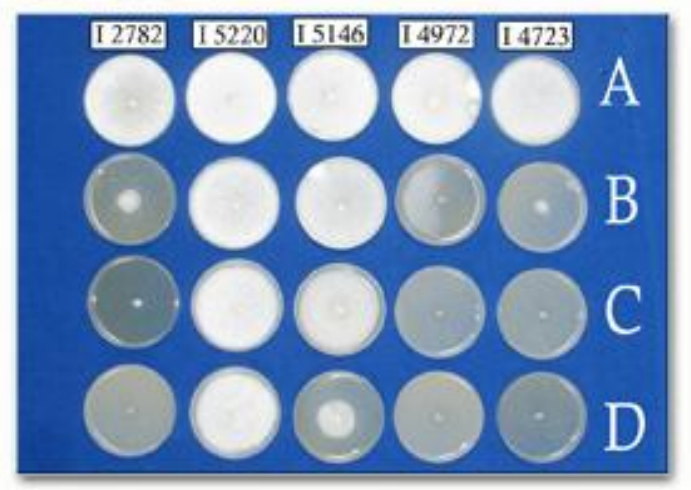

Hexaconazole

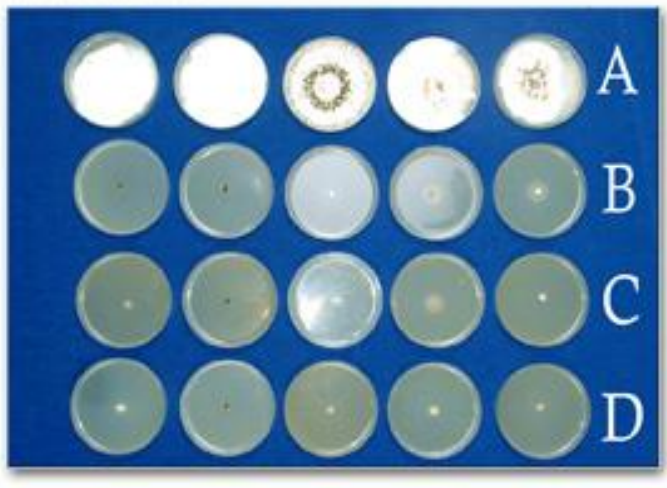

Difenoconazole

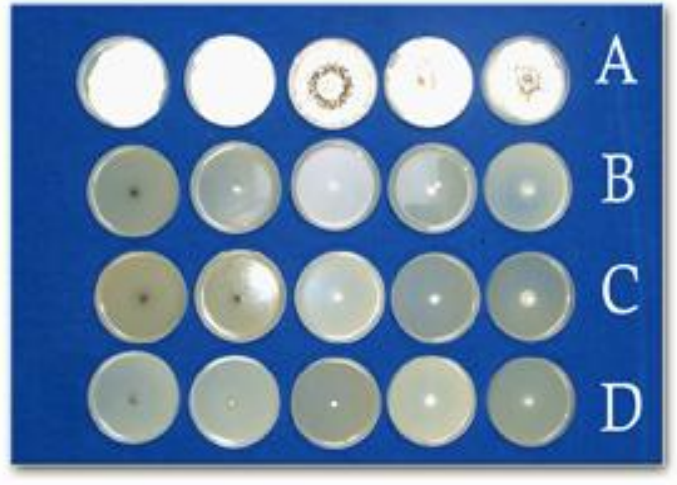

\section{Plate 5: Effect of systemic fungicides on slow} growing isolates of Sclerotium rolfsii.

$$
\text { Concentrations : } \begin{array}{ll}
A=0 \mathrm{ppm} & \begin{array}{l}
\mathrm{E}=250 \mathrm{ppm} \\
B=125 \mathrm{ppm}
\end{array} \\
\mathbf{D}=500 \mathrm{ppm}
\end{array}
$$




\section{Mencozeb}

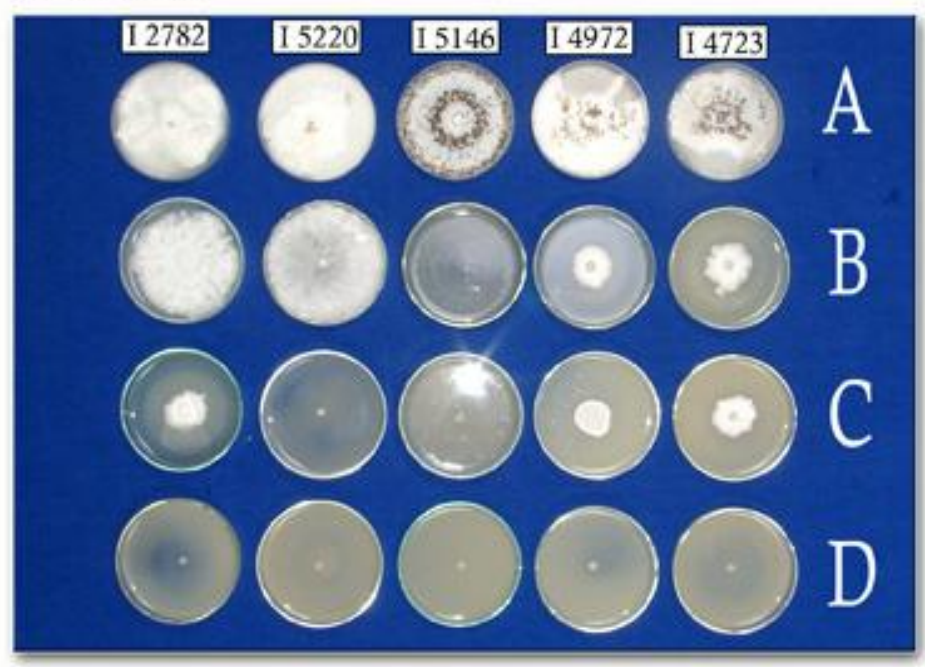

\section{Ghlorothalonil}

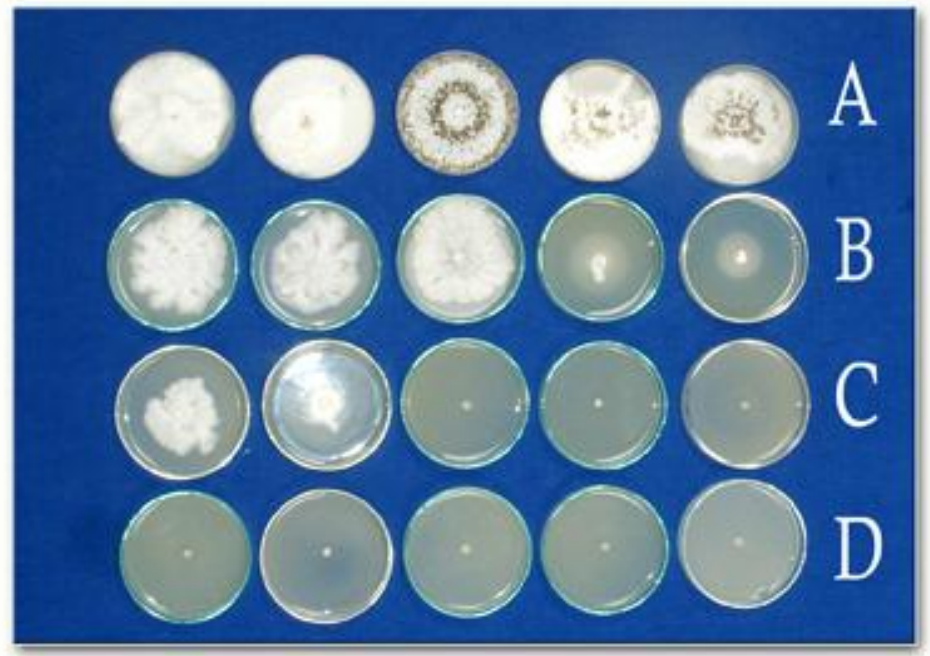

\section{Plate 6: Effect of non - systemic fungicides on} slow growing isolates of Sclerotium rolfsii.

$$
\text { Concentrations : } \begin{aligned}
& A=0 \mathrm{ppm} \\
& B=500 \mathrm{ppm} \\
& C=1000 \mathrm{pm} \\
& D=2000 \mathrm{ppm}
\end{aligned}
$$


Effect of systemic fungicides on slow growing isolates of Sclerotium rolfsii

The poisoned food technique was employed for testing efficacy of systemic fungicides on slow growing isolates of $S$. rolfsii. The observations on the growth of the fungal isolates in each treatment including the check were taken and per cent inhibition was calculated.

Data regarding the per cent inhibition of the fungal growth of isolates are presented in Table 15, 16, 17 and depicted in Plate 5.

The mean inhibition per cent due to various treatments is given in Table 15. It is seen from the data presented in Table 15 that all main effects and interaction effects were observed significant at five per cent level of significance. All isolates differed significantly and the lowest mean inhibition was observed in I $5220(66.67 \%)$ followed by I $5146(68.22$ $\%)$; whereas, the maximum inhibition was observed in I $2782(95.82 \%)$ followed by I $4972(87.70 \%)$ and I $4723(86.59 \%)$. Out of three, two fungicides (hexaconazole and difenoconazole) have indicated cent per cent inhibition in cases of isolates I 5220 and I 2782. However, it was zero per cent inhibition in case of isolate I 5220 followed by I 5146 $(15.44 \%)$ with fungicide carbendazim. Over all comparison of fungicides revealed that the lowest mean inhibition was observed for carbendazim (58.78\%); whereas, remaining two fungicides had shown comparatively more inhibition i.e. 93.89 and 90.33 per cent in case of hexaconazole and difenconazole, respectively. The higher dose of fungicides comparatively gave more inhibition (Table 16). Data pertaining to higher order interaction (I x F x D) presented in Table 17 showed that except isolates I 5220 at all concentrations and I 5146 at $125 \mathrm{ppm}$ concentration of carbendazim; the growth of all isolates was inhibited by fungicides.
The fungicide hexaconazole was the most effective and gave cent per cent growth inhibition for all concentrations tested barring isolates I 4972 and I 4723 (125 ppm). Difenconazole was the next effective fungicide. Isolates I 5220 and I 2782 were completely inhibited by difenoconazole at all concentrations tested.

\section{Effect of non-systemic fungicides on slow growing isolates of Sclerotium rolfsii}

The relative efficacy of non-systemic fungicides on slow growing isolates of $S$. rolfsii in vitro was tested by using poisoned food technique. The fungicides were tested at 500, 1000, $2000 \mathrm{ppm}$ concentrations. Data on the growth of the isolates were taken. The per cent inhibition was calculated on the basis of the differences in growth of isolates observed in the treatment and the check. The growth inhibition per cent of the fungal isolates at various concentrations of fungicides are presented in Table 18, 19, 20 and depicted in Plate 6.

It is seen from Table 18 that all main effects as well as the interaction effects were found significant at five per cent level of significance. The minimum mean per cent inhibition was found in isolate I $2782(44.00$ $\%$ ); whereas, the maximum mean per cent inhibition was observed in isolate I 5146 $(83.33 \%)$. Isolate I 5146 showed cent per cent growth inhibition; whereas, the minimum inhibition $(41.22 \%)$ was observed in I 2782 with mancozeb. In case of chlorothalonil, isolate I 2782 showed minimum inhibition $(46.78 \%)$ and the maximum was in isolate I $4723(88.33 \%)$. Increase in the concentration of fungicides increased the growth inhibition and complete growth inhibition was observed at $2000 \mathrm{ppm}$ dose of both the fungicides. Similar trend was also noticed in case of I x D interactions where cent per cent growth inhibition was noticed at $2000 \mathrm{ppm}$ for all 
isolates (Table 19). Higher order interaction of I x D x F presented in Table 20 indicated that per cent inhibition was increased with increase in the concentration of fungicides. At $2000 \mathrm{ppm}$, the growth of the all isolates was inhibited cent per cent. The isolate I 5146 and I 5220 had not exhibited growth inhibition for chlorothalonil and mancozeb respectively at 500 ppm concentration.

Considering the effect of fungicides on growth of different isolates of $S$. rolfsii, it has been proved in present investigation that hexaconazole and difenoconazole were superior with their effectiveness followed by carbendazim in systemic fungicide. Several researchers have reported the effectiveness of systemic fungicides for growth inhibition of S. rolfsii (Chowdary et al., 1998; Hanumantha and Kannan, 2002; Bhat and Srivastava, 2003; Cilliers et al., 2003; Prabhu and Hiremath, 2003; Thakur et al., 2004). Difenconazole and hexaconazole have completely inhibited growth of all medium growing isolates of the $S$. rolfsii. More or less, similar results were also reported by Johnson and Subramanyam (2000). They investigated the efficacy of eight fungicides against $S$. rolfsii in vitro. Complete inhibition of radial growth of $S$. rolfsii colonies was achieved by hexaconazole. Das et al., (2014), also tested six systemic fungicides, among them hexaconazole was found highly effective in suppressing radial expansion as well as per cent inhibition of $S$. rolfsii. Cent per cent inhibition was observed using 100 ppm concentration at $120 \mathrm{hrs}$ after inoculation. Results proved authentically that hexaconazole treated plants greater accumulate the defense enzymes chitinase, $\beta$ 1, 3-glucanase, and pyrocatechol (an antifungal phenolic), which reduced the disease incidence significantly. It is also proved biochemically and immunologically that such type of induction associated with synthesis of pathogenesis-related PR proteins
PR-2 and PR-3 (Sharma and Chakraborty, 2005). Further, fast, medium and slow growing isolates of $S$. rolfsii showed quite variable results against systemic fungicide carbendazim in present investigation.

Isolates showed tolerant or sensitive reaction to the fungicide. The growth was nil in seven fast growing isolates (I 4743, I 4877, I 5061, I 5518, J 2004 I, J 2004 II and M 1999) and one each from medium and slow growing isolates I 5068 and I 5220, respectively. Johnson and Subramanyam (2000) observed that no inhibition of $S$. rolfsii was recorded with carbendazim. These results are also corroborating the results obtained by Solunke et al., (2001). They investigated the effect of carbendazim against four isolates (SRP-1, SRP-2, SRP-3 and SRP-4) of S. rolfsii and reported that SRP-4 appeared to be tolerant, while SRP-1 was sensitive.

Out of the two non-systemic fungicides evaluated for different isolates of $S$. rolfsii; mancozeb was more effective than chlorothalonil. Effectiveness of non-systemic fungicides against $S$. rolfsii isolated from different host was also reported earlier (Hennriquez and Motealegre, 1992; Chowdary et al., 1998; Bhoraniya et al., 2002; Hanumantha and Kannan, 2002; Das et al., 2014). Slow growing isolates of $S$. rolfsii were completely inhibited at $2000 \mathrm{ppm}$ concentration of mancozeb and chlorothalonil. These results are in confirmation with previously work reported by Chowdary et al., (1998). They noted that non systemic fungicide mancozeb completely inhibited the mycelial growth of $S$. rolfsii at 1000 ppm. Bhat and Srivastava (2003) also observed complete mycelial growth inhibition at $500 \mathrm{ppm}$ of mancozeb. Das et al., (2014) tested three non-systemic fungicides in vitro against $S$. rolfsii, among them mancozeb showed higher inhibitory effect as compared to others. 
Application of fungicides is a part of integrated disease management. We can reduce disease incidence to use of suitable fungicides alone or combinations with their appropriate formulation. Five fungicides at three different concentrations were screened in vitro by poisoned food technique to evaluate their efficacy against different isolates of $S$. rolfsii. All the fungicides tested effectively inhibited the growth of $S$. rolfsii isolates. Among these fungicides, hexaconazole and difenconazole (systemic) were superior at $125 \mathrm{ppm}$ and proved the most effective for suppressing the growth of $S$. rolfsii isolates followed by mancozeb and chlorothalonil (non-systemic). The effectiveness of hexaconazole is already been reported by earlier workers and it is also reported to help plant in accumulation of defence enzymes in plants against pathogen and synthesis of PR-proteins. Carbendazim was not so much effective alone but it was effective with other suitable combinations reported by earlier studies. Mancozeb and chlorothalonil were also effective significantly against $S$. rolfsii isolates.

\section{References}

Amma, P. A. and Shanmugam, N. 1976. Site of infection in sclerotial root of groundnut. Madras Agric. J. 63: 129.

Aycock, R. 1966. Stem rot and other diseases caused by Sclerotium rolfsii. N.C. Agric. Exp. Stn. Tech. Bull. Raleigh, NC, USA. 174: pp 202.

Bhat, N. M, and Srivastava, L. S. 2003. Evaluation of some fungicides and neem formulations against six soilborne pathogens and three Trichoderma spp. in vitro. Plant Dis. Res. 18 (1): 5659.

Bhoraniya, M. F., Khandar, R. R. and Khunti, J. P. 2002. Evaluation of pesticides against Sclerotium rolfsii in chilli by soil plate technique. Plant Dis. Res. 17 (1): 145-146.

Chohan, J. S. 1974. Recent advances in diseases of groundnut in India. In: Current Trends in Plant Pathology (Eds. Raychoudhury, S. P. and Verma, J. P.), Deptt. Bot. Univ., Lucknow, pp 179.

Chowdary, K. A., Reddy, D. R. and Rao, K. C. 1998. Efficacy of systemic (triazoles) and non-systemic fungicides against sclerotial wilt of bell pepper caused by Sclerotium rolfsii Sacc. Indian $\mathrm{J}$. Pl. Protec. 26 (2): 125-130.

Cilliers, A. J. Pretorius, Z. A. and Wyk, P. S. 2003. Integrated control of Sclerotium rolfsii on groundnut in South Africa. $J$. Phytopathol. 151 (5): 249-258.

Das, N. C., Dutta, B. K. and Ray, D. C. 2014. Potential of some fungicides on the growth and development of Sclerotium rolfsii Sacc. In Vitro. International Journal of Scientific and Research Publications, 4 (12) ISSN: 2250-3153.

Desai, S. and Ghewande, M. P. 1991. Incidence of Sclerotium rolfsii on groundnut foliage. Int. Arachis Newsl. No.10.

Dhingra, O. D. and Sinclair, J. B. 1993. Basic Plant Pathology Methods. CBS Publisher \& Distributors, New Delhi, (232 p.) pp 355.

Gazaway, W. S. and Hagan, A. K. 1989. Sclerotium blight. In: Compedium of Soybean Diseases ( $3^{\text {rd }}$ ed.), (Eds. by Sinclair, J. B. and Backman, P. A.), APS Press, St. Paul. pp 48-49.

Hanumantha, B. T. and Kannan, N. 2002. In vitro evaluation of fungicides against soft rot of coffee caused by Sclerotium rolfsii. J. Coffee Res. 30 (2): 137-140.

Henriquez S. J. and Montealegre A. J. 1992. Chemical control of Sclerotium rolfsii Sacc. Agricultura Tecnica (Chile) 52 (1): 79-84.

Johnson, M and Reddy, P.N and Reddy, D. R. 2008. Comparative efficacy of 
rhizosphere mycoflora, fungicides, insecticides and herbicides against groundnut stem rot caused by Sclerotium rolfsii. Ann. Pl. Protec. Sciences. 16 (2): 414-418.

Johnson, M. and Subramanyam, K. 2000. Invitro efficacies of fungicides against stem rot pathogen (Sclerotium rolfsii) of groundnut. Ann. Pl. Protec. Sciences 8 (2): 255-257.

Mayee, C. D. and Datar, V. V. 1988. Diseases of groundnut in the Tropics. Rev. Tropic. Pl. Pathol. 5: 85-118.

Prabhu, H. V. and Hiremath, P. C. 2003. Bioefficacy of fungicides against collar rot of cotton caused by Sclerotium rolfsii Sacc. Karnataka J. Agric. Sciences 16 (4): 576-579.

Punja, Z. K. 1985. The biology, ecology and control of Sclerotium rolfsii. Annu. Rev. Phytopathol. 23: 97-127.

Punja, Z. K. 1988. Sclerotium (Athelia) rolfsii, a pathogen of many plant species. in: Advances in Plant Pathology. Vol.6, Genetics of Plant Pathogenic Fungi. G. S. Sidhu, Academic Press, London. pp 523-534.

Punja, Z. K. and Grogan, R. G. 1983. Hyphal interactions and antagonism among field isolates and single basidiospore strains of Athelia (Sclerotium) rolfsii. Phytopathol. 73: 1279-1284.
Rangaswami, R. 2002. A Text Book of Agricultural Statistics. New Age International (P) Limited, New Delhi, India, pp 496.

Rolfs, P. H., 1892. Tomato blight some hints, Bulletin of Florida Agricultural Experimental Station, pp.18.

Sharma, M. and Chakraborty, B. 2005. Hexaconazole and calixin mediated defense strategies of tea plants against Exobasidium vexans. J. Mycol. Pl. Pathol. 35 (3): 417-429.

Shew, B. B., Beute, M. K. and Campbell, C. L. 1984. Spatial pattern of southern stem rot caused by Sclerotium rolfsii in six North Carolina peanut fields. Phytopathol. 74: 730-735.

Solunke, B. S., Kareppa, B. M. and Gangawane, L. V. 2001. Integrated management of Sclerotium rot of potato using carbendazim and plant extracts. Indian J. Pl. Protec. 29 (1/2): 142-143.

Thakur, K. S., Keshry, P. K., Tamrakar, D. K. and Sinha, A. K. 2004. Studies on management of collar rot disease (Sclerotium rolfsii) of chickpea by use of fungicides. Advances in Plant Sciences 17 (2): 553-555.

Vincent, J. M. 1947. Distortion of fungal hyphae in the presence of certain inhibitors. Nature 159: 850.

\section{How to cite this article:}

Sharma, R.K. and Dhruj, I.U. 2018. In vitro Evaluation of Some Fungicides against Indian Isolates of Sclerotium rolfsii Sacc. [Teleomorph: Athelia rolfsii (Curzi) Tu \& Kimbrough]. Int.J.Curr.Microbiol.App.Sci. 7(11): 1561-1586. doi: https://doi.org/10.20546/ijcmas.2018.711.179 